\title{
OPEN Effect of photobiomodulation therapy on painful temporomandibular disorders
}

\author{
Adila Aisaiti ${ }^{1}$, Yanli Zhou ${ }^{1}$, Yue Wen ${ }^{1}$, Weina Zhou ${ }^{1,2}$, Chen Wang ${ }^{1,2}$, Jing Zhao ${ }^{1,2}$, \\ Linfeng $\mathrm{Yu}^{1,2 \bowtie}$, Jinglu Zhang ${ }^{1,2 \bowtie}$, Kelun Wang ${ }^{2,3,4}$ \& Peter Svensson ${ }^{4,5,6}$
}

To evaluate the effect of photobiomodulation therapy (PBMT) on painful temporomandibular disorders (TMD) patients in a randomized, double-blinded, placebo-controlled manner. Participants were divided into a masseter myalgia group $(n=88)$ and a temporomandibular joint (TMJ) arthralgia group $(n=87)$ according to the Diagnostic Criteria for Temporomandibular Disorders (DC/TMD). Both groups randomly received PBMT or placebo treatment once a day for 7 consecutive days, one session. The PBMT was applied with a gallium-aluminum-arsenide (GaAIAs) laser (wavelength $=810 \mathrm{~nm}$ ) at pre-determined points in the masseter muscle $\left(6 \mathrm{~J} / \mathrm{cm}^{2}, 3\right.$ regions, $\left.60 \mathrm{~s}\right)$ or TMJ region $\left(6 \mathrm{~J} / \mathrm{cm}^{2}, 5\right.$ points, $30 \mathrm{~s}$ ) according to their most painful site. Pain intensity was rated on a $\mathbf{0}-10$ numerical rating scale (NRS) and pressure pain thresholds (PPT) and mechanical sensitivity mapping were recorded before and after the treatment on day 1 and day 7 . Jaw function was assessed by pain free jaw opening, maximum unassisted jaw opening, maximum assisted jaw opening, maximum protrusion and right and left excursion. Data were analyzed with a mixed model analysis of variance (ANOVA). Pain intensity in arthralgia patients decreased over time $(P<0.001)$ for both types of interventions, however, $P B M T$ caused greater reduction in pain scores than placebo $(P=0.014)$. For myalgia patients, pain intensity decreased over time $(P<0.001)$ but without difference between interventions $(P=0.074)$. PPTs increased in both myalgia $(P<0.001)$ and TMJ arthralgia patients over time $(P<0.001)$ but without difference between interventions $(P \geq 0.614)$. Overall, PBMT was associated with marginally better improvements in range of motion compared to placebo in both myalgia and arthralgia patients. Pain intensity, sensory function and jaw movements improve after both PBMT and placebo treatments in myalgia and arthralgia patients indicating a substantial non-specific effect of PBMT.

Temporomandibular disorders (TMD) cover a number of clinical conditions affecting the temporomandibular joint (TMJ), masticatory muscles and related structures ${ }^{1}$. Pain is one of the most common and limiting clinical manifestations of such disorders ${ }^{2}$. The pathophysiology of pain in TMD patients is still not fully understood, however, peripheral and central sensitization of the trigeminal nociceptive pathways in addition to impairment in endogenous pain modulatory mechanisms influenced by genetic and epigenetic factors, and a wide and multifaceted range of environmental conditions such as for example bruxism, psychosocial distress, mood, sleep patterns etc. are encapsulated in the bio-psycho-social model for chronic pain ${ }^{3,4}$. Recent classification schemes have taken the step to include TMD pain as a primary type of chronic pain meaning that pain in itself has become the disease without a clear and identifiable cause whereas the secondary types of (TMD) pain are viewed as painful conditions which are caused by a disease or disorder and therefore represent a symptom rather than a disease ${ }^{5,6}$. In this scenario, causal treatment is not an option and palliative and non-invasive treatment modalities should be preferred. Several non-invasive treatment modalities have been used to relieve painful TMD conditions including occlusal splints, ultrasonography, pharmacologic-based therapy ${ }^{7}$, manipulative therapy ${ }^{8}$, oral motor

\footnotetext{
${ }^{1}$ Department of Orofacial Pain \& TMD, Affiliated Hospital of Stomatology, Nanjing Medical University, 1 Shanghai Road,Gulou District, Nanjing 210029, Jiangsu, People's Republic of China. ${ }^{2}$ Jiangsu Key Laboratory of Oral Diseases, Orofacial Pain \& TMD Research Unit, Affiliated Hospital of Stomatology, Nanjing Medical University, Nanjing, People's Republic of China. ${ }^{3}$ Center for Sensory-Motor Interaction (SMI), Department of Health Science \& Technology, Aalborg University, Ålborg, Denmark. ${ }^{4}$ Section of Orofacial Pain and Jaw Function, Department of Dentistry and Oral Health, Aarhus University, Aarhus, Denmark. ${ }^{5}$ Faculty of Odontology, Malmø University, Malmö, Sweden. ${ }^{6}$ Scandinavian Center for Orofacial Neurosciences (SCON), Aarhus, Denmark. ${ }^{\varpi}$ email: 905151803@ qq.com; zhjllym@njmu.edu.cn
} 


\section{Flowchart}

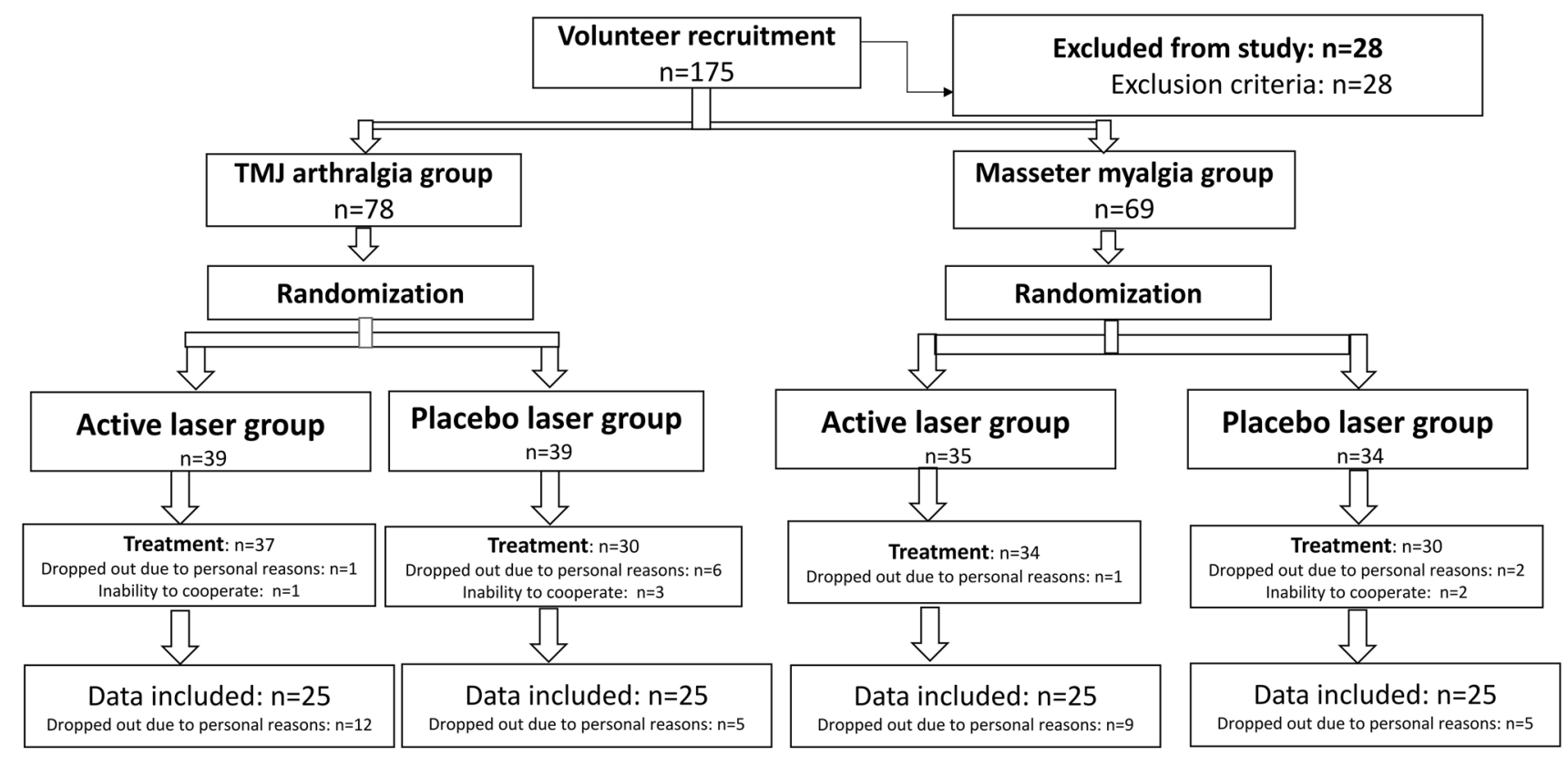

Figure 1. Flow chart of study. Personal reasons: include lack of time, work problem or reluctance to continue the experiment.

exercise $^{9}$, transcutaneous electrical stimulation, and photobiomodulation therapy $(\mathrm{PBMT})^{10}$. Studies in specific TMD pain populations are, however, needed before strong recommendations can made.

PBMT has been widely used as an alternative or complementary treatment for TMD pain due to the noninvasive and safe features related to the low-intensity energy and wavelength characteristics ${ }^{11,12}$. The mechanism of photobiomodulation (PBM) is still debated but may be related to its potential influence on the synthesis, release, and metabolism of countless signaling substances involved in pain and analgesia ${ }^{13,14}$. Many prospective clinical trials have been performed to evaluate the efficacy of PBMT ${ }^{11,13,15-17}$, however, the results have been ambiguous due to the different laser parameters, lack of standardized operation procedures, lack of reliable methodology including placebo control, and small sample sizes. Therefore, more solid evidence is needed to evaluate the therapeutic effect of PBMT in TMD pain patients.

A standardized quantitative sensory testing (QST) developed by the German Research Network on Neuropathic Pain (DFNS) has been shown to be reliable in the orofacial region and upper and lower limbs ${ }^{18-20}$. The QST technique has the advantage of more objective and accurate evaluation of patients' somatosensory function. In addition, a mechanical sensitivity mapping technique which has recently been used in the field of headache disorders such as migraine ${ }^{21}$ and chronic tension-type headache ${ }^{22}$ has been confirmed to have excellent reliability to demonstrate changes in somatosensory function and mechanical sensitivity in the masseter muscle and TMJ regions ${ }^{23}$.

Patients with TMJ pain often involve disorders of the articular surfaces, articular disk, bilaminar zone, joint capsule and ligaments, manifested as sound, limited mandibular movement and pain in these areas, while patients with muscle disorders often present with pain in the related muscle region, reduction of mandibular mobility and "pulling" sensations such as feeling of fatigue and bracing ${ }^{24}$. In addition to these differences in clinical manifestations, the mechanism of TMJ pain and myalgia may also be different ${ }^{24}$. Since there are different clinical manifestations and potentially different underlying pain mechanisms between TMD myalgia and TMJ arthralgia, studies may need to test the efficacy of interventions in both groups to obtain a better platform for evidenced-based recommendations for management. Therefore, the purpose of this study was to evaluate the effect of PBMT on myalgia and TMJ arthralgia patients by use of QST and pain sensitivity mapping techniques in a randomized, double-blinded, placebo-controlled manner.

\section{Results}

A total of 175 participants were initially enrolled into the study, 28 of them were excluded due to the exclusion criteria. 147 participants were randomized into 4 groups, while 16 participants dropped out due to personal reasons or inability to cooperate. In total, 100 participants completed all sessions in the study and 31 participants were lost in the follow-up during the study due to personal reasons (Fig. 1 (Supplementary Fig. S1)). Eight participants (massester myalgia: 3; TMJ arthralgia: 5) from PBMT group received occlucal splint therapy or pharmacology treatment for further treatment after experiment, while all participants in the placebo group received one week of real laser therapy for free, and 3 of them (masseter myalgia: 1; TMJ arthralgia: 2) received occlucal splint therapy at a later stage. No adverse effects were observed during and after the treatment period. The clinical characteristics of participants in each group are shown in Table 1. Regarding the treatment expectations (PBM/ 


\begin{tabular}{|c|c|c|c|c|c|c|c|c|c|c|c|}
\hline \multirow[b]{2}{*}{ Group } & \multirow[b]{2}{*}{ Intervention } & \multicolumn{2}{|l|}{ Age } & \multicolumn{2}{|c|}{ Gender } & \multirow{2}{*}{$\begin{array}{l}\text { Pain intensity (NRS: } \\
0-10)\end{array}$} & \multicolumn{3}{|c|}{ Pain history } & \multicolumn{2}{|c|}{ Pain characteristics } \\
\hline & & Mean & SD & Men & Women & & $\leq 1$ month & 1-6 month & $\geq 6$ month & Intermittent & Persistent \\
\hline \multirow{2}{*}{$\begin{array}{l}\text { Masseter } \\
\text { myalgia }\end{array}$} & PBM & 34.8 & 11.4 & 6 & 19 & 5.3 & 10 & 6 & 9 & 13 & 12 \\
\hline & Placebo & 33.2 & 13.6 & 8 & 17 & 4.4 & 6 & 13 & 6 & 16 & 9 \\
\hline \multirow{2}{*}{ TMJ arthralgia } & PBM & 35.0 & 12.2 & 4 & 21 & 5.2 & 8 & 7 & 10 & 15 & 10 \\
\hline & Placebo & 34.8 & 13.2 & 6 & 19 & 4.1 & 7 & 10 & 8 & 13 & 12 \\
\hline
\end{tabular}

Table 1. Clinic characteristics of participants in different groups. PBM: photobiomodulation.

placebo: mean \pm standard deviation = myalgia: $6.1 \pm 2.4 / 5.4 \pm 2.5$; arthralgia: $6.9 \pm 1.9 / 6.4 \pm 1.7)$, no significant difference was found between $\mathrm{PBM}$ and placebo (myalgia: $\mathrm{F}=1.058, \mathrm{P}=0.314$; arthralgia: $\mathrm{F}=0.933, \mathrm{P}=0.344$ ).

Effect of PBMT on myalgia patients compared with placebo. The relative changes of pressure pain thresholds (PPT) and numerical rating scale (NRS) scores of patients are shown in Fig. 2a (Supplementary Fig S2a). For the pain intensity, the main effect of time was significant for both PBMT and placebo treatment $(\mathrm{F}=30.088 ; \mathrm{P}<0.001)$ without any difference between interventions $(\mathrm{F}=3.492 ; \mathrm{P}=0.074)$, and no significant interactions between time and intervention $(\mathrm{F}=2.551 ; \mathrm{P}=0.104)$; For pressure pain thresholds, PPTs at the masseter muscle and TMJ regions were significantly increased after both PBMT and placebo (Left TMJ: $F=28.893$, $\mathrm{P}<0.001$; Right TMJ: $\mathrm{F}=14.810, \mathrm{P}<0.001$; Left masseter: $\mathrm{F}=27.427, \mathrm{P}<0.001$; Right masseter: $\mathrm{F}=27.488$, $\mathrm{P}<0.001$ ) without significant difference between intervention, (All ANOVAs: $\mathrm{F}<2.544 ; \mathrm{P}>0.124$ ) and no significant interactions between time and intervention was found (All ANOVAs: $\mathrm{F}<2.542 ; \mathrm{P}>0.063$ ).

For mechanical sensitivity mapping, the changes of the mean NRS values of each grid point in every examination are presented in Fig. 3 (Supplementary Fig. S3). There were significant main effects of time on center of gravity (COG) coordinates and entropy for both PBMT and placebo treatment (COG-X: $\mathrm{F}=15.639, \mathrm{P}<0.001, \mathrm{COG}-\mathrm{Y}$ : $\mathrm{F}=16.029, \mathrm{P}<0.001$, Entropy: $\mathrm{F}=7.893, \mathrm{P}<0.001)$, but no significant main effects of intervention (COG-X: $\mathrm{F}=0.718, \mathrm{P}=0.405$; COG- $\mathrm{Y}: \mathrm{F}=0.631, \mathrm{P}=0.435$; Entropy: $\mathrm{F}=0.416, \mathrm{P}=0.525$ ) nor interactions between time and intervention were found (COG-X: $\mathrm{F}=1.815, \mathrm{P}=0.177$; $\mathrm{COG}-\mathrm{Y}: \mathrm{F}=1.115, \mathrm{P}=0.336$; Entropy: $\mathrm{F}=1.726, \mathrm{P}=0.185$ ).

The results of analysis regarding jaw function assessment including pain free jaw opening, maximum unassisted jaw opening, maximum assisted jaw opening, maximum protrusion excursion and right and left excursion, COG coordinates and entropy of myalgia patients are shown in Table 2. For jaw movements, the participants in the PBMT group showed more improvement in mouth-opening and lateral excursion movement than those in the placebo group from Day 7-pre (pain free jaw opening: $\mathrm{F}=4.919, \mathrm{P}=0.046$, maximum unassisted jaw opening: $\mathrm{F}=7.466, \mathrm{P}=0.022$, maximum assisted jaw opening: $\mathrm{F}=5.706, \mathrm{P}=0.021$, left lateral excursion: $\mathrm{F}=2.688, \mathrm{P}=0.016$, right lateral excursion: $\mathrm{F}=3.018, \mathrm{P}=0.041)$ and significant interaction effects between time and intervention were found in mouth-opening (pain free jaw opening: $\mathrm{F}=5.191, \mathrm{P}=0.024$, maximum unassisted jaw opening: $\mathrm{F}=6.106, \mathrm{P}=0.012$, maximum assisted jaw opening: $\mathrm{F}=6.150, \mathrm{P}=0.008)$ and lateral excursion movements of patients( left lateral excursion: $\mathrm{F}=7.826, \mathrm{P}=0.001$, right lateral excursion: $\mathrm{F}=3.839, \mathrm{P}=0.024$ ).

Effect of PBMT on TMJ arthralgia patients compared with placebo. The relative changes of PPTs and NRS scores of patients are shown in Fig. 2b (Supplementary Fig S2b). For pain intensity, both groups showed a significant reduction over time $(\mathrm{F}=25.469, \mathrm{P}<0.001)$. The NRS scores of patients were significantly lower in the PBMT group compared to the placebo group $(\mathrm{F}=6.998, \mathrm{P}=0.014)$, but no significant interaction between time and intervention was found $(\mathrm{F}=1.045, \mathrm{P}=0.350)$; For Pressure pain thresholds, the main effects of time were found in the PPTs of the right TMJ, left masseter muscle and right masseter muscle (right TMJ: $F=17.009$, $\mathrm{P}<0.001$, left masseter: $\mathrm{F}=29.878, \mathrm{P}<0.001$, right masseter: $\mathrm{F}=14.356, \mathrm{P}<0.001$ ) without significant differences between intervention (All ANOVAs: $\mathrm{F}<0.261$; $\mathrm{P}>0.614$ ) or interaction between time and intervention (All ANOVAs: $\mathrm{F}<1.129 ; \mathrm{P}>0.337)$. The PPTs in the left TMJ increased over time $(\mathrm{F}=16.080, \mathrm{P}<0.001)$, but no significant treatment effect was found between the $\mathrm{PBMT}$ and placebo groups $(\mathrm{F}=0.010, \mathrm{P}=0.923)$, however, the interaction between time and intervention was statistically significant $(\mathrm{F}=3.753, \mathrm{P}=0.015)$.

For mechanical sensitivity mapping, the changes of the mean NRS values of each grid in every examination are shown in Fig. 4 (Supplementary Fig. S4). Entropy values significantly decreased over time following both interventions $(\mathrm{F}=91.749, \mathrm{P}<0.001)$ and were significantly lower after PBM compared to placebo at Day 7-post $(\mathrm{F}=3.179, \mathrm{P}=0.041)$. A significant interaction effect between time and intervention of entropy was also found in the TMJ arthralgia patients $(\mathrm{F}=3.347, \mathrm{P}=0.048)$. In terms of the COG coordinates, the COGX and COGY were significantly decreased over time (COGX: $\mathrm{F}=15.369, \mathrm{P}<0.001, \mathrm{COGY}: \mathrm{F}=21.877, \mathrm{P}<0.001$ ) without a treatment difference (COGX: $\mathrm{F}=0.268, \mathrm{P}=0.609$; COGY: $\mathrm{F}=0.116, \mathrm{P}=0.737$ ) or interaction effect between time and intervention (COGX: $\mathrm{F}=0.354, \mathrm{P}=0.651$; $\mathrm{COGY}: \mathrm{F}=0.462, \mathrm{P}=0.573$ );

The results of analysis regarding jaw function assessment, COG coordinates and entropy of TMJ arthralgia patients are shown in Table 3. For jaw movements, the ranges of pain free jaw opening and right lateral excursion improved over time following both types of intervention (pain free jaw opening: $\mathrm{F}=28.793, \mathrm{P}<0.001$; right lateral excursion: $\mathrm{F}=4.911, \mathrm{P}=0.013$ ) while no significant main effect of intervention or interactions between time and intervention were found (All ANOVAs: $\mathrm{F}<2.618, \mathrm{P}>0.101$ ). Significant improvements in maximum unassisted jaw opening over time were found following both types of interventions $(\mathrm{F}=10.034, \mathrm{P}=0.001)$, but more improvement was shown in the PBMT group compared to the placebo group from Day 1-post $(\mathrm{F}=10.565$, 


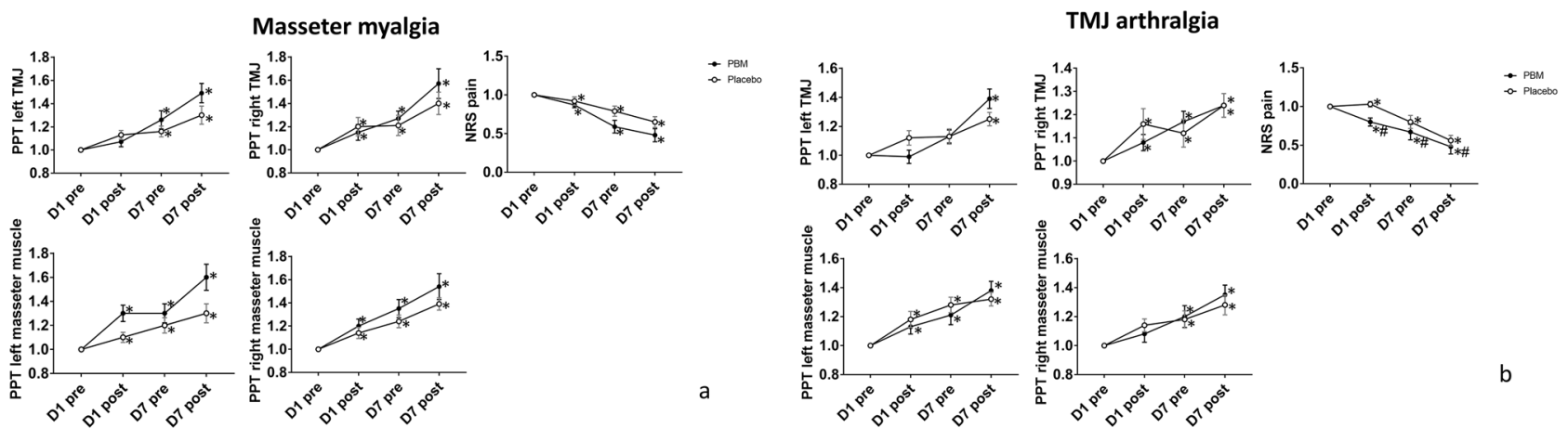

Figure 2. (a) The relative change of pressure pain thresholds (PPT) and NRS pain scores (mean and standard error) in masseter myalgia patients during the experiment $(n=50)$. Photobiomodulation (PBM) group $(n=25)$; Placebo group $(\mathrm{n}=25)$. “*” indicates significant difference between $\mathrm{D} 1$-pre and other time points $(\mathrm{P}<0.05) .(\mathbf{b})$ The relative change of pressure pain thresholds (PPT) and NRS pain scores (mean and standard error) in TMJ arthralgia patients during the experiment $(n=50)$. Photobiomodulation $(P B M)$ group $(n=25)$; Placebo group $(\mathrm{n}=25)$. " " indicates significant difference between D1-pre and other time points $(\mathrm{P}<0.05)$; “\#” indicates significant difference between $\mathrm{PBM}$ and placebo $(\mathrm{P}<0.05)$.

$\mathrm{P}=0.041)$. No significant improvements were shown in maximum assisted jaw opening, maximum protrusion nor left lateral excursion movement following any of the interventions (All ANOVAs: $\mathrm{F}<2.479, \mathrm{P}>0.113$ ).

\section{Discussion}

This randomized, placebo-controlled study indicated that both PBM and placebo interventions can reduce the self-reported pain intensity and improve the jaw function in TMD pain patients, but PBM showed marginally better effectiveness in patients with TMJ arthralgia and more advantages in improving jaw movement compared to placebo. Pain sensitivity of TMD patients appears to improve over time following both PBMT and placebo treatment without specific effects or differences between subgroups of TMD pain.

Putative effects of PBMT on pain. As a non-invasive and non-pharmacological modality, PBMT has been suggested to be effective in improving mandibular movements and promoting analgesia in TMD pain patients ${ }^{10,13,25-32}$. The hypothesis on PBM effects mainly covers three aspects: modulation of inflammatory processes, alteration of excitation and nerve conduction in peripheral nerves, and release of endogenous endorphins. The effects of PBMT on inflammatory processes include changes in biochemical markers (histamine, prostaglandin E2, interleukin-1, tumor necrosis factor- $\beta$ ), altered distributions of inflammatory cells, reduced formation of edema, hemorrhage, and necrosis ${ }^{33}$. The thinly myelinated A- $\sigma$ and unmyelinated, slow-conducting $\mathrm{C}$ fiber distributed under the epidermis form the peripheral nerve endings of nociceptors can be activated by noxious stimuli leading to propagation of action potentials. When the laser energy is absorbed by the skin, these action potentials are believed to be inhibited, causing changes in activation threshold of the nerve fibers and a decrease in the release of pro-inflammatory neuropeptides (i.e., substance $\mathrm{P}$ ) ${ }^{34}$. The increase of serotonin and endorphin levels is a further putative mechanism of pain relief $\mathrm{f}^{35}$. Although these putative molecular and biological mechanisms underlying of PBMT is far from complete, it is commonly believed that Cytochrome $\mathrm{c}$ Oxidase (CCO) in the mitochondrial respiratory chain serves as the primary photoceptor ${ }^{36}$. Since the action spectrum of PBMT matches the absorption spectrum of CCO, after absorption of photons by CCO, inhibitory Nitric Oxide (NO) which is non-covalently bound to the heme and $\mathrm{Cu}$ centers of $\mathrm{CCO}$ and competitively blocks oxygen at a ratio of 1:10 can be dissociated, and the activation of mitochondrial respiration can lead to increase of mitochondrial membrane potential (MMP), cyclic adenosine monophosphate (cAMP), oxygen resumption and ATP production ${ }^{37}$. NO is known as a vasodilator, which can stimulate the soluble guanylate cyclase to form cyclic-GMP (cGMP) and activates protein kinase G, which leads to reuptake of $\mathrm{Ca}^{2+}$ and opening of calciumactivated potassium channels, causing the relaxation of blood vessels and lymphatic vessels ${ }^{38}$. The mediated activation of transient receptor potential (TRP) ion-channels is also considered as one of the mechanisms of action of PBM. After laser irradiation, these cation channels will open and depolarize the cell membrane, the increase of intracellular $\mathrm{Ca}^{2+}$ can lead to the release of histamine to promote tissue healing ${ }^{39,40}$. Besides that, the lipid peroxidation of cell membranes can also lead to a generation of reactive oxygen species (ROS) ${ }^{41}$. ROS can activate the transcription factor nuclear factor kappa $B(N F-k B)$, which regulates the expression of genes related cellular functions such as inflammatory-induced response and survival ${ }^{42}$. The activation of NF-kB can be induced by PBM to enhance gene transcription that leads to cell proliferation and migration, to reduce cell death and enhance neurological function ${ }^{37}$. Although the evidence for the mechanism of action of PBM is mounting, there continues to be controversies about the clinical effectiveness of PBMT in TMD pain patients due to lack of standardization of laser parameters and operating procedures, as well as lack of high-quality and sufficiently operationalized outcome measures. Therefore, the present study applied reliable and validated QST measures and comprehensive pain mapping techniques to provide more objective and robust evidence to evaluate the therapeutic effect of PBMT stimulation. 

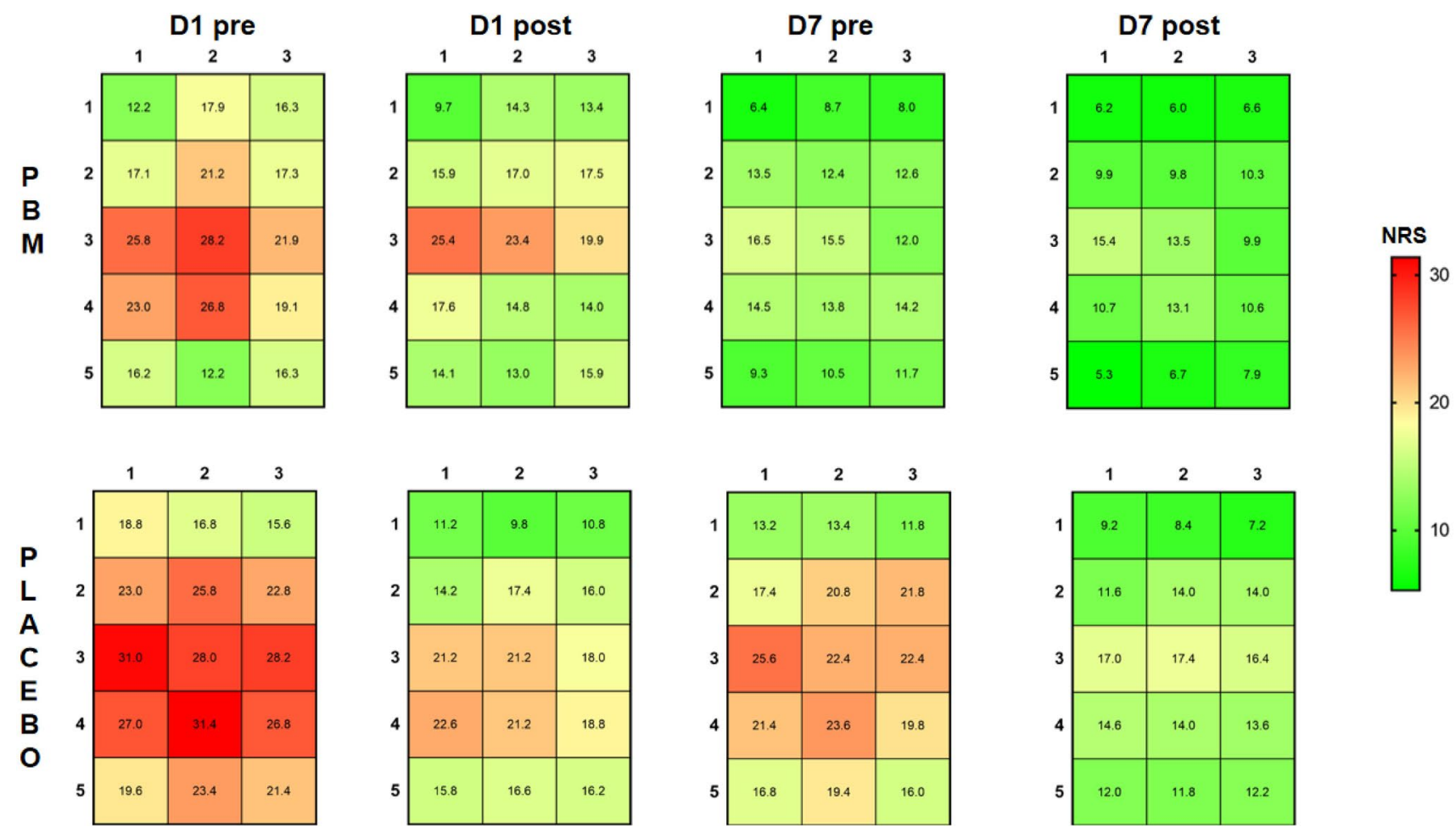

Figure 3. The mechanical sensitivity mapping of masseter myalgia patients before and after the treatment with photobiomodulation $(\mathrm{PBM})(\mathrm{n}=25)$ and placebo $(\mathrm{n}=25)$. NRS: numerical rating scale from $0-50-100$ with $50=$ moderate painful. Measurements were taken before and after treatment at Day 1 and Day 7 (D1 pre/D1 post; D7 pre/D7 post).

\begin{tabular}{|c|c|c|c|c|c|c|c|c|c|}
\hline \multirow[b]{2}{*}{ Parameter } & \multirow[b]{2}{*}{ Intervention } & \multicolumn{2}{|c|}{ D1pre } & \multicolumn{2}{|c|}{ D1post } & \multicolumn{2}{|l|}{ D7pre } & \multicolumn{2}{|c|}{ D7post } \\
\hline & & Mean & SD & Mean & SD & Mean & SD & Mean & SD \\
\hline \multicolumn{10}{|l|}{ Jaw movement } \\
\hline \multirow{2}{*}{ Pain free jaw opening (mm) } & PBM & 28.0 & 6.9 & $29.5^{*}$ & 7.5 & $33.7^{* *}$ & 9.0 & $34.8^{* *}$ & 8.2 \\
\hline & Placebo & 34.4 & 5.2 & 35.8 & 5.3 & 35.3 & 5.8 & 36.6 & 5.4 \\
\hline \multirow{2}{*}{ Maximum unassisted jaw opening (mm) } & PBM & 34.5 & 7.4 & $36.2^{*}$ & 7.4 & $37.6^{* *}$ & 7.9 & $38.8^{* *}$ & 7.8 \\
\hline & Placebo & 40.2 & 4.7 & 41.2 & 5.2 & 40.3 & 5.4 & 41.1 & 4.8 \\
\hline \multirow{2}{*}{ Maximum assisted jaw opening (mm) } & PBM & 40.4 & 5.4 & 40.7 & 5.5 & $42.4^{*}$ & 5.5 & $43.0^{* *}$ & 5.3 \\
\hline & Placebo & 42.3 & 4.7 & 42.5 & 4.5 & 41.9 & 4.1 & 42.5 & 3.8 \\
\hline \multirow{2}{*}{ Protrusion excursion (mm) } & PBM & 5.2 & 2.5 & 5.6 & 2.5 & 5.7 & 2.4 & $6.0^{*}$ & 2.5 \\
\hline & Placebo & 4.6 & 2.1 & 5.0 & 1.8 & 4.3 & 1.8 & $4.8^{*}$ & 1.7 \\
\hline \multirow{2}{*}{ Left lateral excursion (mm) } & PBM & 8.2 & 2.2 & 8.0 & 2.3 & $9.0^{\#}$ & 2.3 & $9.4^{* \#}$ & 2.0 \\
\hline & Placebo & 8.2 & 2.8 & 8.6 & 2.8 & 7.7 & 2.7 & 8.3 & 2.7 \\
\hline \multirow{2}{*}{ Right lateral excursion (mm) } & PBM & 6.8 & 3.2 & 7.0 & 2.7 & $8.1^{\#}$ & 2.4 & $8.8^{* \#}$ & 2.5 \\
\hline & Placebo & 7.8 & 2.4 & 8.4 & 2.4 & 7.9 & 2.8 & 8.6 & 2.4 \\
\hline \multicolumn{10}{|l|}{ Mechanical sensitivity mapping } \\
\hline \multirow{2}{*}{ COGX } & PBM & 1.2 & \begin{tabular}{|l|}
0.9 \\
\end{tabular} & $1.1^{*}$ & 1.0 & 1.0 & \begin{tabular}{|l|}
0.9 \\
\end{tabular} & $0.7^{\star}$ & \begin{tabular}{|l|}
0.7 \\
\end{tabular} \\
\hline & Placebo & 1.4 & 1.2 & $1.1^{*}$ & 1.0 & 1.4 & 1.2 & $0.9^{*}$ & 0.8 \\
\hline \multirow{2}{*}{ COGY } & PBM & 1.9 & 1.5 & $1.6^{*}$ & 1.5 & 1.6 & 1.3 & $1.1^{*}$ & 1.0 \\
\hline & Placebo & 2.2 & 1.8 & $1.7^{*}$ & 1.7 & 2.2 & 1.9 & $1.4^{*}$ & 1.3 \\
\hline \multirow{2}{*}{ Entropy } & PBM & 0.9 & 0.6 & 0.9 & 0.6 & 0.8 & 0.6 & $0.6^{*}$ & 0.6 \\
\hline & Placebo & 1.0 & 0.5 & 0.8 & 0.5 & 0.9 & 0.6 & $0.7^{*}$ & 0.4 \\
\hline
\end{tabular}

Table 2. The descriptive statistics (mean \pm standard deviation) and results of analysis regarding the test parameters of the masseter myalgia group during the experiment $(n=50)$. Photobiomodulation $(P B M)$ group $(\mathrm{n}=25)$; Placebo group $(\mathrm{n}=25)$; “»” indicates significant difference between D1-pre and other time points $(\mathrm{P}<0.05)$; “\#” indicates significant differences between PBM and placebo $(\mathrm{P}<0.05)$. 

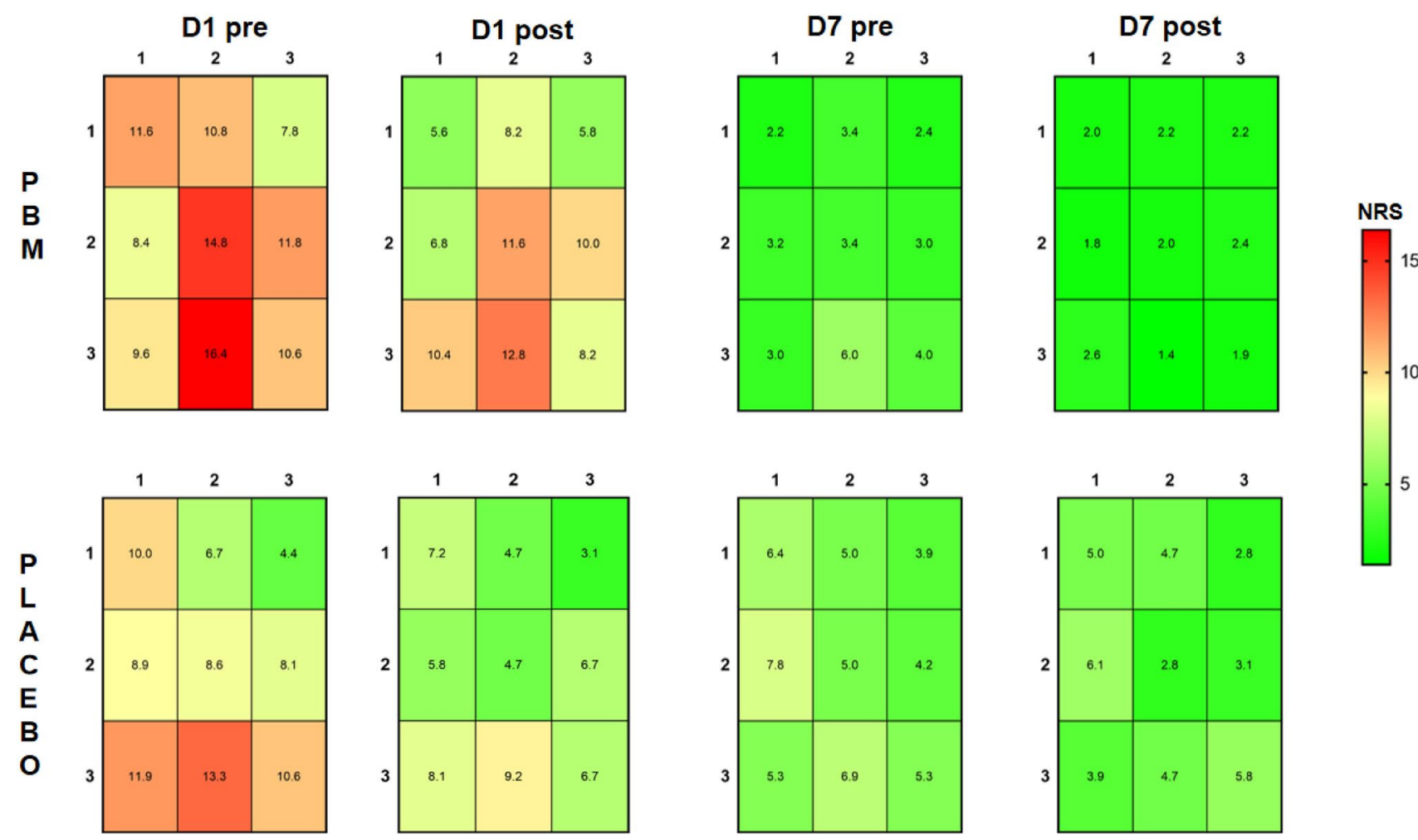

Figure 4. The mechanical sensitivity mapping of TMJ arthralgia patients before and after the treatment with photobiomodulation $(\mathrm{PBM})(\mathrm{n}=25)$ and placebo $(\mathrm{n}=25)$. NRS: numerical rating scale from $0-50-100$ with $50=$ moderate painful. Measurements were taken before and after treatment at Day 1 and Day 7 (D1 pre / D1 post; D7 pre/D7 post).

\begin{tabular}{|c|c|c|c|c|c|c|c|c|c|}
\hline \multirow[b]{2}{*}{ Parameter } & \multirow[b]{2}{*}{ Intervention } & \multicolumn{2}{|l|}{ D1pre } & \multicolumn{2}{|c|}{ D1post } & \multicolumn{2}{|l|}{ D7pre } & \multicolumn{2}{|c|}{ D7post } \\
\hline & & Mean & SD & Mean & SD & Mean & SD & Mean & SD \\
\hline \multicolumn{10}{|l|}{ Jaw movement } \\
\hline \multirow{2}{*}{ Pain free jaw opening $(\mathrm{mm})$} & PBM & 30.3 & 8.3 & $32.7^{*}$ & 7.2 & $36.8^{*}$ & 5.8 & $38.4^{*}$ & 6.3 \\
\hline & Placebo & 33.0 & 6.9 & $35.4^{*}$ & 6.3 & $36.4^{*}$ & 6.5 & $37.8^{*}$ & 5.6 \\
\hline \multirow{2}{*}{ Maximum unassisted jaw opening (mm) } & PBM & 38.4 & 6.3 & $39.9^{\#}$ & 5.9 & $41.1^{* \#}$ & 5.2 & $42.1^{\star \#}$ & 5.4 \\
\hline & Placebo & 40.8 & 4.9 & 40.8 & 5.4 & 40.8 & 5.2 & 41.2 & 4.8 \\
\hline \multirow{2}{*}{ Maximum assisted jaw opening (mm) } & PBM & 42.2 & 5.4 & 43.4 & 5.1 & 43.8 & 3.8 & 44.1 & 4.1 \\
\hline & Placebo & 42.6 & 3.7 & 42.6 & 3.6 & 43.0 & 2.9 & 43.3 & 3.0 \\
\hline \multirow{2}{*}{ Protrusion excursion (mm) } & PBM & 5.0 & 2.2 & 5.1 & 1.8 & 5.2 & 2.0 & 5.6 & 1.9 \\
\hline & Placebo & 5.5 & 2.0 & 5.7 & 1.8 & 5.6 & 0.8 & 5.8 & 0.9 \\
\hline \multirow{2}{*}{ Left lateral excursion (mm) } & PBM & 8.3 & 2.2 & 8.7 & 2.5 & 8.8 & 2.1 & 9.0 & 2.5 \\
\hline & Placebo & 7.7 & 3.2 & 7.9 & 3.3 & 7.4 & 3.2 & 8.0 & 3.1 \\
\hline \multirow{2}{*}{ Right lateral excursion (mm) } & PBM & 7.8 & 2.9 & 8.5 & 2.2 & 8.8 & 2.1 & $9.2^{*}$ & 1.8 \\
\hline & Placebo & 7.4 & 3.1 & 7.6 & 3.3 & 8.2 & 2.7 & $8.6^{*}$ & 2.7 \\
\hline \multicolumn{10}{|l|}{ Mechanical sensitivity mapping } \\
\hline \multirow{2}{*}{ COGX } & PBM & 2.0 & 0.5 & 2.1 & 0.4 & $1.7^{\star}$ & 1.0 & $1.5^{*}$ & 1.2 \\
\hline & Placebo & 1.9 & 0.4 & 1.9 & 0.4 & $1.3^{*}$ & 0.9 & $1.3^{*}$ & 0.9 \\
\hline \multirow{2}{*}{ COGY } & PBM & 2.1 & 0.6 & 2.1 & 0.4 & $1.7^{\star}$ & 1.0 & $1.4^{*}$ & 1.1 \\
\hline & Placebo & 2.4 & 0.4 & 2.4 & 0.5 & $1.6^{*}$ & 1.1 & $1.6^{*}$ & 1.1 \\
\hline \multirow{2}{*}{ Entropy } & PBM & 0.5 & 0.5 & $0.4^{*}$ & 0.4 & $0.2^{*}$ & 0.3 & $0.1^{* \#}$ & 0.3 \\
\hline & Placebo & 0.5 & 0.5 & $0.4^{*}$ & 0.5 & $0.3^{*}$ & 0.4 & $0.3^{*}$ & 0.4 \\
\hline
\end{tabular}

Table 3. The descriptive statistics (mean \pm standard deviation) and results of analysis regarding the test parameters of the TMJ arthralgia group during the experiment $(n=50)$. Photobiomodulation $(P B M)(n=25)$; Placebo $(\mathrm{n}=25)$; “*” indicates significant difference between D1-pre and other time points $(\mathrm{P}<0.05)$; “\#” indicates significant differences between $\mathrm{PBM}$ and placebo $(\mathrm{P}<0.05)$. 
Laser parameter. The effectiveness of PBM is related to the laser irradiation parameters including wavelength, power, power density, energy density, time and total energy ${ }^{35}$. In previous studies, it has been shown that lower doses of light are more effective than much higher doses due to a biphasic dose response curve exhibited by $\mathrm{PBM}^{43,44}$. In clinical practice, multiple PBM treatment protocols are available for TMD patients, after reviewing all available therapeutic regimens, a recent systematic review suggested an evidence-based protocol for clinical PBM administration for these patients ${ }^{45}$. According to this review, the application of GaAlAs diode laser, wavelength 800-900 nm, 100-500 $\mathrm{mW}$ and energy density lower than $10 \mathrm{~J} / \mathrm{cm}^{2}$, twice a week for 30 days showed the best results for pain relief and improvement of mandibular movements ${ }^{45}$. To achieve the same total dose as the recommendation ${ }^{45}$ and minimize the possibility of spontaneous remission, we modified the treatment application to once a day for 7 consecutive days.

Outcome measures. In many previous studies on PBMT in TMD pain patients the focus has been on jaw movements or self-reported pain intensity, only a few studies have determined changes in patients' somatosensory function using more objective testing methods ${ }^{46,47}$. For example, simple rating scales like a NRS or VAS (Visual Analogue Scale) are the most popular way to evaluate the self-reported pain intensity in clinical practice; however, this approach may be an oversimplification of complex biopsychosocial pain problems and possibly result in an underestimation or overestimation of pain ${ }^{46}$.

Pressure algometry is part of the QST technology and is the most-commonly used method to detect pressure sensitivity and static mechanical allodynia in deep tissues ${ }^{19}$. Pressure algometry delivers both a stable, reliable and quantifiable pressure through a flat base applied to the dermal surface ${ }^{48}$. The force is generally applied as a gradual increased pressure stimulus by the examiner, and the stimulation is stopped once the test participant presses a button. The value of interest is typically defined as the pressure pain threshold (PPT) which is minimum pressure that the participant report to be consistently just barely painful ${ }^{49,50}$. PPT has been shown to be reliable for evaluation of deep pain sensitivity in e.g., the masseter muscle and TMJ regions ${ }^{18-20}$. In addition, a pain mapping technique has also been demonstrated to have excellent reliability in assessment of the spatial aspects of mechanical sensitivity in the masseter muscle area and TMJ regions ${ }^{23}$. This method is considered as a valuable approach for a more comprehensive investigation of the pathology of somatosensory system, and it can provide an overall description of how somatosensory function varies with time or change in sensitivity among multiple test sites $^{51}$. Therefore, the pain mapping technique, which was performed with a simple quantitative palpometer in this study, is one of the main novelties of this experiment and demonstrated significant changes in deep pain sensitivity across the masseter and TMJ regions following both PBM and placebo interventions while PBM showed more advantage than placebo in TMJ arthralgia patients.

Effects of PBMT on masseter myalgia. The present study showed that the self-reported pain intensity in patients with masseter myalgia was decreased and the somatosensory sensitivity was improved (less sensitive) following the intervention with both PBM and placebo, which are consistent with previous studies ${ }^{52,53}$. The present study found that the jaw movement of PBMT group showed more improvement than those in the placebo group, which is also corroborated by other studies ${ }^{54-56}$. The effectiveness of placebo in this study can also be related to the activation of endogenous opioids and neural mechanisms of pain modulation ${ }^{57-59}$. Pain modulation is, indeed, influenced by many factors, such as psychological issues, memory of pain experience, hope of health restoration, educational level and professional-patient relationship etc. ${ }^{60-62}$. The sham-laser treatment provided in the placebo sessions may have amplified the psychological impact and anticipation of treatment effects in myalgia patients which is reflected in similar expectations scores between PBM and placebo. Furthermore, a good relationship between the professionals and patients in the present experiment may also contribute to the improvement following the placebo intervention. From this perspective, we can consider that although the therapeutic effect of PBMT has been indicated in animal studies ${ }^{63,64}$, in human-beings the cognitive aspects of the placebo intervention is as important as the putative biological effects following PBMT in mediating an hypoalgesia effect in TMD pain patients with a sub-diagnosis of myalgia.

As for the results of pain mapping in myalgia patients, it was clear that patients showed the highest NRS scores around the middle part of the masseter muscle, while the lowest NRS value was found near bony structures and in the periphery of the masseter muscle consistent with previous studies ${ }^{23}$. Interestingly, the NRS pain scores decreased, COG coordinates shifted, and entropy values decreased as a reflection of a decrease and more homogenous muscle pain sensitivity following both types of interventions. This finding again questions the specificity of PBMT in patients with myalgia.

Effects of PBMT on TMJ arthralgia. In the present study, PBM was found to have some minor advantages compared to placebo in terms of decreases in self-reported pain intensity and increases in maximum unassisted jaw opening as well as improvement in mechanical sensitivity in patients with TMJ arthralgia. However, in Emshoff et al's study ${ }^{17}$, the self-reported pain relief following PBM and placebo were both decreased but with no significant difference between groups. It should also be acknowledged that the present differences between $\mathrm{PBM}$ and placebo are small and perhaps without a robust clinical importance. Similar to the findings in myalgia patients, the pain mapping results indicated lower entropy scores following PBMT in TMJ arthralgia suggesting a normalization of the pain sensitivity in the TMJ region. It can be speculated that this effect is mediated by a combination of endogenous pain modulation mechanisms related to a placebo response and a peripheral component related to the putative therapeutic effect of PBMT on peripheral sensitization of the TMJ region. Direct measures of the biological markers in the TMJ would add more information to the understanding of PBMT effects in future studies. 
Limitations of the study. First, it is important to notice that the standardized operating procedure in the scientific literature about the dose and protocols of PBMT for TMD pain patients has not reached a consensus, however, we followed the best available evidence in terms of stimulation parameters. The present results cannot be extrapolated to all types of PBMT for different types of TMD pain patients. Second, it should also be mentioned that only the immediate effect of PBMT was evaluated in this study and the sample size was relatively small partly because of dropouts during the treatment procedure-a well-known challenge in RCT studies. We recommend that multicenter studies are performed in well-defined TMD patient groups (e.g., in accordance with the ICOP criteria) and with larger sample size and reliable and valid outcome measures including the proposed techniques from this study. Nevertheless, it should also be mentioned that we applied rigorous diagnostic criteria to subtype TMD pain patients and this is the first study to investigate PBMT in both TMJ arthralgia and myalgia patients.

\section{Conclusions}

A striking finding in the present experiment was that both PBM and placebo interventions were associated with significant improvement over time and with only subtle differences between myalgia and arthralgia patients. PBMT seemed slightly more effective in terms of a decrease in self-reported pain intensity and improvement of the mechanical sensitivity in patients with TMJ arthralgia while PBM was more effective in terms of improvement of jaw movements in patients with masseter myalgia. Overall, these findings suggest that specific pain management should be tailored to the different subtype of TMD pain and that PBMT may involve substantial placebo effects.

\section{Materials and methods}

Study participants. A total of 175 TMD pain patients (68 men and 107 women, aged from 18 to 60 years old) were involved into the study from April 20th, 2020 to September 30th, 2020. The patients were recruited from the Department of TMD \& Orofacial pain, Affiliated Hospital of Stomatology, Nanjing Medical University, P. R. China. The sample size was calculated a priori based on the detection of a minimum clinically relevant difference of $25 \%$ at an a level of 0.05 and $80 \%$ power (i.e., the risk of a type I and type II error was $5 \%$ and $20 \%$, respectively). However, considering an anticipated $25 \%$ dropout rate, a total of 131 participants were involved and 100 patients were included for data analysis. All patients were examined and diagnosed with Diagnostic Criteria for Temporomandibular Disorders (DC/TMD) ${ }^{65}$ by trained examiners and medical histories were collected. Inclusion criteria were: TMD patients aged from 18 to 60 years old reporting unilateral pain in the masseter muscle or TMJ regions lasting at least 2 weeks without receiving alternative treatments in the past 3 months. The pain intensity assessed on a $0-10$ NRS during manual palpation should be $\geq 4$. Exclusion criteria were: history of orofacial trauma that affects normal somatosensory function; history of serious or chronic systemic diseases (e.g., trigeminal neuralgia, burning mouth syndrome or systemic musculoskeletal pain disorders such as fibromyalgia, or symptoms of rheumatoid arthritis); use of alcohol or caffeine within $24 \mathrm{~h}$ of the test day, and/ or any mental disorders.

According to the exclusion criteria, 28 patients were excluded, 78 patients ( 50 women, 28 men; mean age \pm SD: $34.9 \pm 12.7$ years) were recruited into the TMJ arthralgia group, and 69 patients (46 women, 23 men, mean age \pm SD: $34.0 \pm 12.6$ years) were recruited into the masseter myalgia group (Fig. 1 (Supplementary Fig. S1)). Patients in both groups were randomly assigned to be treated with PBM or placebo according to the results of computer randomization program which was put in an opaque envelope. An individual unassociated with the study prepared the envelopes. Randomizations sequence was created using Stata version 9.0 statistical software and was stratified with a 1:1 allocation using block sizes of $4,5,6$.

Study protocol. A randomized, placebo-controlled, double-blinded clinical trial was conducted by two examiners. Examiner 1 was responsible for the examinations of participants before and after treatment and was blinded to which treatment the patient received, while the examiner 2 completed the randomization and treatment. Participants in both groups were randomly assigned to intervention with PBM or placebo once a day for 7 days. A specialist from the department of orofacial pain and TMD monitored the conditions of the participants during the whole study to take effective measures once the adverse effects occur.

All the examinations and treatment were performed in a reserved room with suitable temperature, and free from sound interference. During the treatment session, the participants were seated upright on a comfortable office chair to measure their jaw movement. Then, they were asked to rate the pain intensity with the use of a numerical rating scale $0-10$ (NRS). After that, they were required to lie down in the dental chair, and examiner 1 measured the PPT values bilaterally at the center of the masseter muscle and at the hinge axis points of the TMJ regions (Fig. 5b (Supplementary Fig. S5b)). Pain sensitivity maps over the painful masseter muscle area and TMJ region were also evaluated by use of quantitative palpation ${ }^{23}$. There were 15 test points (Fig. 5a (Supplementary Fig. S5a)) above the superficial masseter muscle while the TMJ region included 9 test points (Fig. 5b (Supplementary Fig. S5b)). The NRS values reported by the patients at the test points after palpation were used as grids values to create a sensitivity map. Besides that, patients' expectations to the allocated treatment were recorded with the use of a $0-10$ NRS with 0 indicating "expectation of no treatment effect" and 10 indicating "expectations of the most imaginable effect". All the above tests were assessed before (Day 1-pre) and after (Day 1 -post) the treatment on day 1 and day 7 (Day 7-pre, Day 7-post).

Laser and placebo treatment. According to the concept of "Optical window" 35 and the evidence-based protocol for PBM administration ${ }^{45}$, a gallium-aluminum-arsenide (GaAlAs) laser (Pilot, CAO group, America) (wavelength $=810 \mathrm{~nm}$ ) was used for PBMT and placebo treatment. The laser application was performed at pre- 

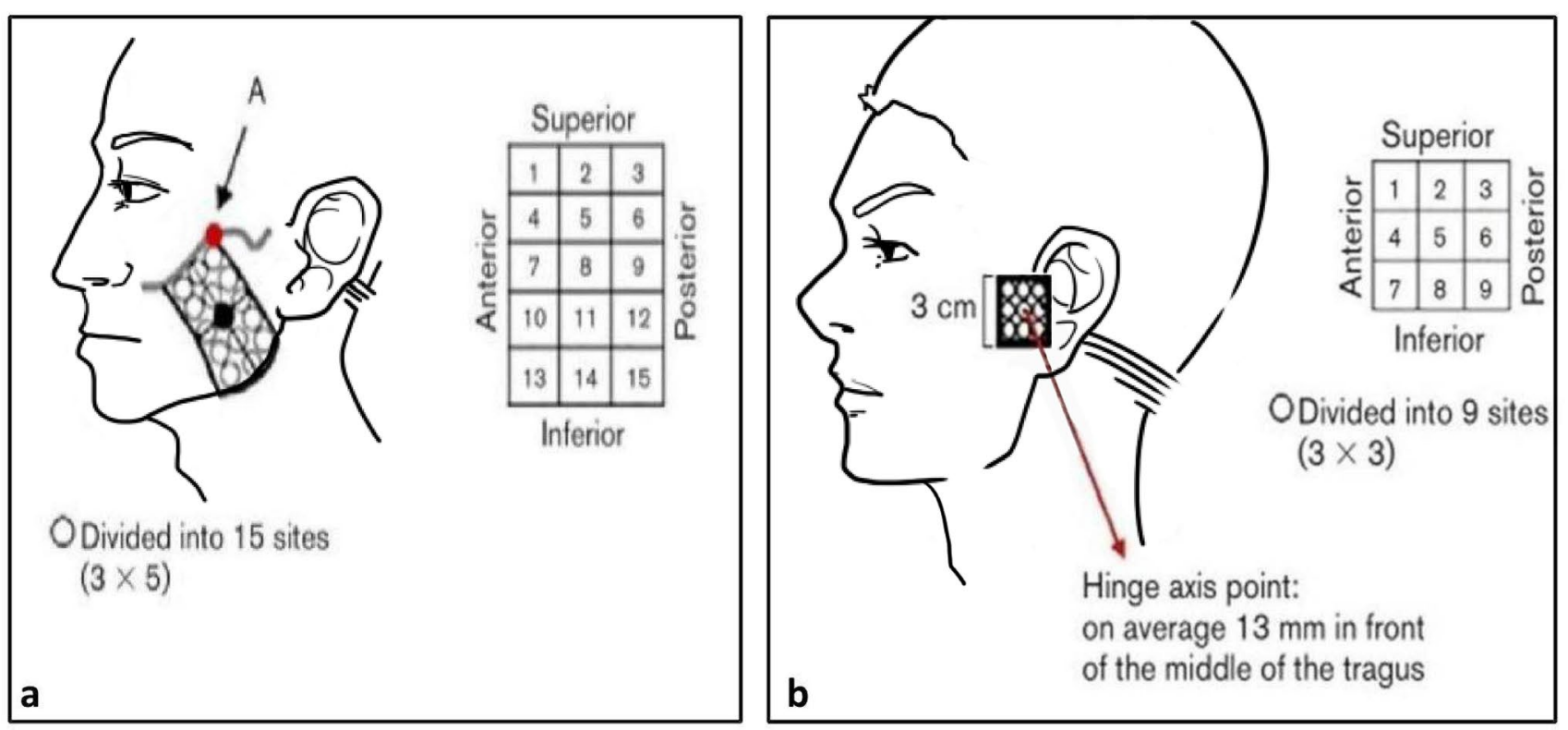

Figure 5. A quantitative mechanical palpometer with 1.0-kg pressure force was applied to 15 grids of masseter muscle (a) and a palpometer with $0.5-\mathrm{kg}$ pressure force was applied to 9 grids of TMJ region (b). Point $\mathrm{A}=$ intersection point between the superior margin and posterior margin of the superficial masseter muscle. In the TMJ region the hinge axis point was on average $13 \mathrm{~mm}$ in front of the middle of the tragus.

determined points on their masseter muscle area or TMJ region of their painful site: the masseter muscle (3 regions: superior, middle, inferior) and TMJ region (4 points forming a cross and one central point) ${ }^{46}$. Since the area of the masseter muscle was different in each individual and limited by the size of the laser light spot, every irradiation region of the masseter muscle was irradiated from anterior to posterior with approximately three to four irradiated circles to ensure all the test points were involved and received equal irradiation. The treatment application was once a day for 7 consecutive days. The PBM stimulation was applied in the continuous emission mode and did not have direct contact with the patient's skin or mucous membrane, and irradiated the treatment site in a circular manner with a special trumpet-shaped handle. The irradiation parameters used were as follows: operating frequency $=10 \mathrm{~Hz}$, laser optical power $=3 \mathrm{~W}$, diameter of light $\mathrm{spot}=2 \mathrm{~cm}$, the distance between spot and optical fiber $=3 \mathrm{~cm}$; for masseter muscle: energy density $=6 \mathrm{~J} / \mathrm{cm}^{2}$, measurements of the output power in irradiated area $=100 \mathrm{~mW}$, time per region $=20 \mathrm{~s}$, total time $=60 \mathrm{~s}$, for the TMJ area: energy density $=6 \mathrm{~J} / \mathrm{cm}^{2}$, measurements of the output power in irradiated area $=100 \mathrm{~mW}$, time per point $=6 \mathrm{~s}$, total time $=30 \mathrm{~s}$. The placebo treatment was done exactly in the same manner as the PBMT at the same points for the same time duration but without power output. During the treatment procedure, both participants and the professional responsible for the treatment were wearing special glasses for eye protection.

Primary outcome measures. Pain intensity recording. Pain intensity was assessed by means of a $0-10$ NRS where 0 indicates "no pain" and 10 means "the most pain imaginable". Patients were instructed to rate the present pain intensity, and the NRS score was used to represent the intensity of patients' pain at the time of evaluation.

Jaw movement measurement. Jaw movement including pain free jaw opening, maximum unassisted jaw opening, maximum assisted jaw opening, maximum protrusion excursion, left lateral excursion, and right lateral excursion were assessed during each examination per the DC/TMD instructions. The distance between the midline of upper and lower central incisors was measured with a ruler.

Secondary outcome measures. Pressure pain thresholds (PPT). PPTs were measured by a hand-held electronic pressure algometer (Algometer; Medoc, Ramat Yishai, Israel) with a probe diameter of $8 \mathrm{~mm}$. The pressure was increased at a rate of $30 \mathrm{kPa} / \mathrm{s}^{66}$. The PPTs were measured bilaterally at the center of the masseter muscle and hinge axis points of the TMJs. Each site was tested for 3 times and the average was calculated and used for the statistical analysis.

Mechanical sensitivity mapping. A quantitative mechanical palpometer (Palpeter, Sunstar Suisses SA) was used to perform standardized palpation of the painful masseter muscle and TMJ regions to form a mechanical sensitivity map and analyze the pain intensity at different and multiple test points. The circular metal rod of the palpometer was $10 \mathrm{~mm}$ in diameter and made of aluminum. There was a hole at the other end of the palpometer to let the stamp-tapering end pass through. The palpometer was held perpendicularly to the surface skin of the 
participants with the examiner's thumb and middle finger. The examiner would detect the tapered end with the index finger as the correct force was applied ${ }^{23}$.

In order to investigate the mechanical sensitivity maps, the superficial part of the masseter muscle was divided into $3 \times 5$ grids (Fig. 5a (Supplementary Fig. S5a)) while the TMJ region was divided into $3 \times 3$ grids (Fig. 5b (Supplementary Fig S5b) $)^{23}$. The palpometer with a $1.0-\mathrm{kg}$ pressure force was applied to each of the 15 grid points at the masseter muscle, and a palpometer with $0.5-\mathrm{kg}$ pressure force was applied to the 9 grid points of the TMJ region in a randomized order in accordance with the (DC/TMD) guidelines for manual palpation. All test sites were stimulated for approximately $2 \mathrm{~s}$ during each measurement. After each measurement, there was a 10 -s interval for the participants to rate the perceived pain intensity of the stimulus on a 0-100 NRS which 0 meant "no pain", 50 was defined "moderate painful", and 100 "the most pain imaginable" 67. The COG coordinates ( $\mathrm{x}=$ anterior-posterior direction and $\mathrm{y}=$ superior-inferior direction) were defined as: $\sum X i *$ grid value $(i) / \sum$ grid value $(i) ; \sum y i *$ grid value $(i) / \sum$ grid value $(i)$; The $0-100$ NRS values were used as grid values ${ }^{68}$. Shannon entropy was also calculated to represent the complexity and diversity of patients' mechanical sensitivity ${ }^{69,70}$.

Data analysis. In order to ensure the baseline was comparable and minimize the impact of patient-related factors such as individual cognitive differences, the way and time of enrollment, the different stages and duration of the disease, all parameters except entropy values, which was presented as the absolute difference (Tn-T0) were otherwise expressed as relative changes from baseline (Day 1-pre) (TN/T0) and logarithmic transformed to normalize the data distribution. Then, the calculated absolute difference and relative change from baseline were used for data analysis. A mixed model analysis of variance (ANOVA) was used to assess the main effects of time (repeated factor), intervention (between group factor) and interactions between factors. A significance level of $5 \%$ was applied.

All statistical work was performed using the Statistical Package for Social Sciences version 20 (SPSS, IBM). The figures and tables presented in this study were produced by Prism 7.0 software.

Ethics approval and consent to participate. All procedures performed in studies were in accordance with the 1964 Helsinki declaration and its later amendments or comparable ethical standards. Confirms that informed consent was obtained from all participants and/or their legal guardians. The study was approved by the Nanjing Medical University Research Ethics Committee (PJ2019-005-01), and registered on the WHO international clinical trial registry platform (20/04/2020, ChiCTR2000032104).

Consent to publish. Confirms informed consent was obtained to publish the information in an online open access publication.

\section{Data availability}

The datasets generated during and/or analyzed during the current study are available from the corresponding author on reasonable request.

Received: 27 October 2020; Accepted: 25 March 2021

Published online: 27 April 2021

\section{References}

1. Carrasco, T. G., Mazzetto, M. O., Mazzetto, R. G. \& Mestriner, W. Jr. Low intensity laser therapy in temporomandibular disorder: A phase II double-blind study. Cranio 26(4), 274-281 (2008).

2. Herpich, C. M. et al. Analysis of laser therapy and assessment methods in the rehabilitation of temporomandibular disorder: A systematic review of the literature. J. Phys. Ther. Sci. 27(1), 295-301 (2015).

3. Chichorro, J. G., Porreca, F. \& Sessle, B. Mechanisms of craniofacial pain. Cephalalgia 37(7), 613-626 (2017).

4. Vaegter, H. B., Fehrmann, E., Gajsar, H. \& Kreddig, N. Endogenous modulation of pain: The role of exercise, stress, and cognitions in humans. Clin. J. Pain. 36(3), 150-161 (2020).

5. Monaco, A., Cattaneo, R., Marci, M. C., Pietropaoli, D. \& Ortu, E. Central sensitization-based classification for temporomandibular disorders: A pathogenetic hypothesis. Pain Res. Manag. 2017, 5957076 (2017).

6. Schiffman, E. \& Ohrbach, R. Executive summary of the diagnostic criteria for temporomandibular disorders for clinical and research applications. J. Am. Dent. Assoc. 147(6), 438-445 (2016).

7. Abbasgholizadeh, Z. S., Evren, B. \& Ozkan, Y. Evaluation of the efficacy of different treatment modalities for painful temporomandibular disorders. Int. J. Oral Maxillofac. Surg. 49(5), 628-635 (2020).

8. Amaral, A. P. et al. Immediate effect of nonspecific mandibular mobilization on postural control in subjects with temporomandibular disorder: A single-blind, randomized, controlled clinical trial. Braz. J. Phys. Ther. 17(2), 121-127 (2013).

9. Machado, B. C., Mazzetto, M. O., Da Silva, M. A. \& de Felício, C. M. Effects of oral motor exercises and laser therapy on chronic temporomandibular disorders: A randomized study with follow-up. Lasers Med. Sci. 31(5), 945-954 (2016).

10. Seifi, M. et al. Comparative effectiveness of low level laser therapy and transcutaneous electric nerve stimulation on temporomandibular joint disorders. J. Lasers Med. Sci. 8(Suppl 1), S27-S31 (2017).

11. Venancio, R. A., Camparis, C. M. \& Lizarelli, R. F. Low intensity laser therapy in the treatment of temporomandibular disorders: A double-blind study. J. Oral Rehabil. 32(11), 800-807 (2005).

12. Carvalho, C. M. et al. Wavelength effect in temporomandibular joint pain: A clinical experience. Lasers Med. Sci. 25(2), 229-232 (2010).

13. Marini, I., Gatto, M. R. \& Bonetti, G. A. Effects of superpulsed low-level laser therapy on temporomandibular joint pain. Clin. J. Pain. 26(7), 611-616 (2010).

14. Prindeze, N. J., Moffatt, L. T. \& Shupp, J. W. Mechanisms of action for light therapy: A review of molecular interactions. Exp. Biol. Med. 237(11), 1241-1248 (2012).

15. Ferreira, L. A., de Oliveira, R. G., Guimarães, J. P., Carvalho, A. C. \& De Paula, M. V. Laser acupuncture in patients with temporomandibular dysfunction: A randomized controlled trial. Lasers Med. Sci. 28(6), 1549-1558 (2013). 
16. Conti, P. C. Low level laser therapy in the treatment of temporomandibular disorders (TMD): A double-blind pilot study. Cranio 15(2), 144-149 (1997).

17. Emshoff, R., Bösch, R., Pümpel, E., Schöning, H. \& Strobl, H. Low-level laser therapy for treatment of temporomandibular joint pain: A double-blind and placebo-controlled trial. Oral Surg. Oral Med. Oral Pathol. Oral Radiol. Endod. 105(4), 452-456 (2008).

18. Geber, C. et al. Psychophysics, flare, and neurosecretory function in human pain models: Capsaicin versus electrically evoked pain. J. Pain. 8(6), 503-514 (2007).

19. Pigg, M., Baad-Hansen, L., Svensson, P., Drangsholt, M. \& List, T. Reliability of intraoral quantitative sensory testing (QST). Pain 148(2), 220-226 (2010).

20. Baad-Hansen, L. et al. Reliability of intra-oral quantitative sensory testing (QST) in patients with atypical odontalgia and healthy controls-A multicentre study. J. Oral Rehabil. 42(2), 127-135 (2015).

21. Barón, J. et al. Differences in topographical pressure pain sensitivity maps of the scalp between patients with migraine and healthy controls. Headache 57(2), 226-235 (2017).

22. Fernández-de-las-Peñas, C. et al. Bilateral pressure pain sensitivity mapping of the temporalis muscle in chronic tension-type headache. Headache 48(7), 1067-1075 (2008).

23. Tang, Z. T. et al. Reliability of mechanical sensitivity mapping in the orofacial region of healthy Chinese individuals: Towards standardized assessment of somatosensory function. J. Oral Facial Pain Headache. 32(4), 400-408 (2018).

24. Axel, B. \& Ulrich, L. Syntax of referencing. In TMJ Disorders and Orofacial Pain: The Role of Dentistry in a Multidisciplinary Diagnostic Approach, Vol. 32, No 3, 212 (2003).

25. Brochado, F. T. et al. Comparative effectiveness of photobiomodulation and manual therapy alone or combined in TMD patients: A randomized clinical trial. Braz. Oral Res. 32, e50 (2018).

26. Rodrigues, J. H. et al. Evaluation of pain, jaw movements, and psychosocial factors in elderly individuals with temporomandibular disorder under laser phototherapy. Lasers Med. Sci. 30(3), 953-959 (2015).

27. Ahrari, F., Madani, A. S., Ghafouri, Z. S. \& Tunér, J. The efficacy of low-level laser therapy for the treatment of myogenous temporomandibular joint disorder. Lasers Med. Sci. 29(2), 551-557 (2014).

28. Da Silva, M. M. et al. Randomized, blinded, controlled trial on effectiveness of photobiomodulation therapy and exercise training in the fibromyalgia treatment. Lasers Med. Sci. 33(2), 343-351 (2018).

29. Mazzetto, M. O., Hotta, T. H. \& Pizzo, R. C. Measurements of jaw movements and TMJ pain intensity in patients treated with GaAlAs laser. Braz. Dent. J. 21(4), 356-360 (2010).

30. Magri, L. V., Carvalho, V. A., Rodrigues, F. C. C., Bataglion, C. \& Leite-Panissi, C. R. A. Non-specific effects and clusters of women with painful TMD responders and non-responders to LLLT: Double-blind randomized clinical trial. Lasers Med. Sci. 33(2), 385-392 (2018).

31. Shobha, R., Narayanan, V. S., Jagadish Pai, B. S., Jaishankar, H. P. \& Jijin, M. J. Low-level laser therapy: A novel therapeutic approach to temporomandibular disorder-A randomized, double-blinded, placebo-controlled trial. Indian J. Dent. Res. 28(4), 380-387 (2017).

32. Cavalcanti, M. F. et al. Comparative study of the physiotherapeutic and drug protocol and low-level laser irradiation in the treatment of pain associated with temporomandibular dysfunction. Photomed. Laser Surg. 34(12), 652-656 (2016).

33. Bjordal, J. M., Johnson, M. I., Iversen, V., Aimbire, F. \& Lopes-Martins, R. A. Low-level laser therapy in acute pain: A systematic review of possible mechanisms of action and clinical effects in randomized placebo-controlled trials. Photomed. Laser Surg. 24(2), $158-168$ (2006).

34. Bromm, B. The infrared laser in the diagnosis of normal and disturbed pain pathways. Schmerz 7(4), 195-203 (1993).

35. Cotler, H. B., Chow, R. T., Hamblin, M. R. \& Carroll, J. The use of low-level laser therapy (LLLT) for musculoskeletal pain. MOJ Orthop. Rheumatol. 2(5), 00068 (2015).

36. De Freitas, L. F. \& Hamblin, M. R. Proposed mechanisms of photobiomodulation or low-level light therapy. IEEE J. Sel. Top. Quantum Electron. 22(3), 7000417 (2016).

37. Hamblin, M. R. Mechanisms and mitochondrial redox signaling in photobiomodulation. Photochem. Photobiol. 94(2), 199-212 (2018).

38. Murad, F. Discovery of some of the biological effects of nitric oxide and its role in cell signaling. Biosci. Rep. 24(4-5), 452-474 (2004).

39. Hardie, R. C. Photosensitive TRPs. Handb. Exp. Pharmacol. 223, 795-826 (2014).

40. Yang, W. Z., Chen, J. Y., Yu, J. T. \& Zhou, L. W. Effects of low power laser irradiation on intracellular calcium and histamine release in RBL-2H3 mast cells. Photochem. Photobiol. 83(4), 979-984 (2007).

41. Iua, V., Klebanov, G. I., Borisenko, G. G. \& Osipov, A. N. Molecular and cellular mechanisms of the low intensity laser radiation effect. Biofizika 49(2), 339-350 (2004).

42. Chen, A. C. et al. Low-level laser therapy activates NF-kB via generation of reactive oxygen species in mouse embryonic fibroblasts. PLoS ONE 6(7), e22453 (2011).

43. Woodruff, L. D. et al. The efficacy of laser therapy in wound repair: A meta-analysis of the literature. Photomed. Laser Surg. 22(3), 241-247 (2004).

44. Huang, Y. Y., Chen, A. C., Carroll, J. D. \& Hamblin, M. R. Biphasic dose response in low level light therapy. Dose Response. 7(4), 358-383 (2009).

45. Tunér, J., Hosseinpour, S. \& Fekrazad, R. Photobiomodulation in temporomandibular disorders. Photobiomodul. Photomed. Laser Surg. 37(12), 826-836 (2019).

46. Magri, L. V., Carvalho, V. A., Rodrigues, F. C., Bataglion, C. \& Leite-Panissi, C. R. Effectiveness of low-level laser therapy on pain intensity, pressure pain threshold, and SF-MPQ indexes of women with myofascial pain. Lasers Med. Sci. 32(2), 419-428 (2017).

47. Chen, J., Huang, Z., Ge, M. \& Gao, M. Efficacy of low-level laser therapy in the treatment of TMDs: A meta-analysis of 14 randomised controlled trials. J. Oral Rehabil. 42(4), 291-299 (2015).

48. Yang, G., Baad-Hansen, L., Wang, K., Xie, Q. F. \& Svensson, P. A study on variability of quantitative sensory testing in healthy participants and painful temporomandibular disorder patients. Somatosens Mot. Res. 31(2), 62-71 (2014).

49. Jones, D. H., Kilgour, R. D. \& Comtois, A. S. Test-retest reliability of pressure pain threshold measurements of the upper limb and torso in young healthy women. J. Pain. 8(8), 650-656 (2007).

50. Rolke, R., Andrews, C. K., Magerl, W. \& Treede, R. D. Deep pain thresholds in the distal limbs of healthy human subjects. Eur. J. Pain. 9(1), 39-48 (2005).

51. Thygesen, T. H., Nørholt, S. E., Jensen, J. \& Svensson, P. Spatial and temporal assessment of orofacial somatosensory sensitivity: A methodological study. J. Orofac. Pain. 21(1), 19-28 (2007).

52. Venezian, G. C., da Silva, M. A., Mazzetto, R. G. \& Mazzetto, M. O. Low level laser effects on pain to palpation and electromyographic activity in TMD patients: A double-blind, randomized, placebo-controlled study. Cranio 28(2), 84-91 (2010).

53. Da Cunha, L. A., Firoozmand, L. M., da Silva, A. P., Camargo, S. E. \& Oliveira, W. Efficacy of low-level laser therapy in the treatment of temporomandibular disorder. Int. Dent. J. 58(4), 213-217 (2008).

54. da Silva, M. M. et al. Randoomized, blinded, controlled trial on effectiveness of photobiomodulation therapy and exercise training in the fibromyalgia treatment. Lasers Med. Sci. 33, 343-351 (2018). 
55. Magri, L. V., Carvalho, V. A., Rodrigues, F. C. C., Bataglion, C. \& Leite-Panissi, C. R. A. Non-specific effects and clusters of women with painful TMD responders and non-responders to LLLT: Double-blind randomized clinical trial. Lasers Med. Sci. 33, 385-392 (2018).

56. Shobha, R., Narayanan, V. S., Pai, B. J., Jaishankar, H. \& Jijin, M. Low-level laser therapy: A novel therapeutic approach to temporomandibular disorder-a randomized, double-blinded, placebo-controlled trial. Indian J. Dent. Res. 28, 380 (2017).

57. Benedetti, F., Pollo, A. \& Colloca, L. Opioid-mediated placebo responses boost pain endurance and physical performance: Is it doping in sport competitions?. J. Neurosci. 27(44), 11934-11939 (2007).

58. Wager, T. D. et al. Placebo-induced changes in FMRI in the anticipation and experience of pain. Science 303(5661), 1162-1167 (2004).

59. Eippert, F., Finsterbusch, J., Bingel, U. \& Büchel, C. Direct evidence for spinal cord involvement in placebo analgesia. Science 326(5951), 404 (2009).

60. Lim, P. F., Smith, S., Bhalang, K., Slade, G. D. \& Maixner, W. Development of temporomandibular disorders is associated with greater bodily pain experience. Clin. J. Pain. 26(2), 116-120 (2010).

61. Chouchou, F. \& Lavigne, G. J. Placebo analgesia and sleep. Pathol. Biol. 62(5), 270-275 (2014).

62. Gourion, D. \& Mouchabac, S. Placebo effect: Clinical, biological and therapeutical involvements in depression. Encephale. 42(1 Suppl 1), 1S24-1S30 (2016).

63. Iyomasa, M. M. et al. Zymographic and ultrastructural evaluations after low-level laser irradiation on masseter muscle of HRS/J strain mice. Lasers Med Sci. 28(3), 777-783 (2013).

64. Desiderá, A. C., Nascimento, G. C., Gerlach, R. F. \& Leite-Panissi, C. R. Laser therapy reduces gelatinolytic activity in the rat trigeminal ganglion during temporomandibular joint inflammation. Oral Dis. 21(5), 652-658 (2015).

65. Schiffman, E. et al. Diagnostic criteria for temporomandibular disorders (DC/TMD) for clinical and research applications: Recommendations of the International RDC/TMD consortium network ${ }^{*}$ and orofacial pain special interest group $\dagger$. J. Oral Facial Pain Headache. 28(1), 6-27 (2014).

66. Bisset, L. M., Evans, K. \& Tuttle, N. Reliability of 2 protocols for assessing pressure pain threshold in healthy young adults. J. Manipulative Physiol. Ther. 38(4), 282-287 (2015).

67. Svensson, P., Graven-Nielsen, T. \& Arendt-Nielsen, L. Mechanical hyperesthesia of human facial skin induced by tonic painful stimulation of jaw muscles. Pain 74(1), 93-100 (1998).

68. Ridding, M. C., Brouwer, B., Miles, T. S., Pitcher, J. B. \& Thompson, P. D. Changes in muscle responses to stimulation of the motor cortex induced by peripheral nerve stimulation in human subjects. Exp. Brain Res. 131(1), 135-143 (2000).

69. Shannon, C. E. The mathematical theory of communication. MD Comput. 14(4), 306-317 (1997).

70. Castrillon, E. E. et al. Entropy of masseter muscle pain sensitivity: A new technique for pain assessment. J. Oral Facial Pain Headache. 31(1), 87-94 (2017).

\section{Acknowledgements}

This study was supported by the Orofacial Pain \& TMD Research Unit, Institute of Stomatology, Affiliated Hospital of Stomatology, Nanjing Medical University, Nanjing, P.R. China. The authors declared the study was carried out with no financial funding or insurance support. All the figures and tables shown in the study were made by the author Adila Aisaiti.

\section{Author contributions}

J.Z., L.Y., K.W., P.S. contributed to the study design, data analysis, and editing of the manuscript. A.A., Y.Z., Y.W. contributed to the conducting of study, study design, and data collection. A.A. contributed to the data analysis and drafting of the manuscript. W.Z., C.W., J.Z. contributed to the study supervision, participants collection.

\section{Competing interests}

The authors declare no competing interests.

\section{Additional information}

Supplementary Information The online version contains supplementary material available at https://doi.org/ 10.1038/s41598-021-87265-0.

Correspondence and requests for materials should be addressed to L.Y. or J.Z.

Reprints and permissions information is available at www.nature.com/reprints.

Publisher's note Springer Nature remains neutral with regard to jurisdictional claims in published maps and institutional affiliations.

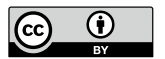

Open Access This article is licensed under a Creative Commons Attribution 4.0 International License, which permits use, sharing, adaptation, distribution and reproduction in any medium or format, as long as you give appropriate credit to the original author(s) and the source, provide a link to the Creative Commons licence, and indicate if changes were made. The images or other third party material in this article are included in the article's Creative Commons licence, unless indicated otherwise in a credit line to the material. If material is not included in the article's Creative Commons licence and your intended use is not permitted by statutory regulation or exceeds the permitted use, you will need to obtain permission directly from the copyright holder. To view a copy of this licence, visit http://creativecommons.org/licenses/by/4.0/.

(C) The Author(s) 2021 